\title{
Biliary cystadenoma - A rare cystic neoplasm of liver
}

\author{
Vrushali V Shroff*, Sandhya V Poflee and Nadia Ilias \\ Department of Pathology, Government Medical College and Hospital, Maharashtra, India
}

\begin{abstract}
Biliary cystadenoma is a rare, benign cystic neoplasm of liver which is seen to occur in intra as well as extrahepatic location. Intrahepatic cystadenomas show a strong tendency to recur particularly in cases with incomplete resection and has a high potential for malignant transformation. It is crucial to differentiate biliary cystadenoma from other cystic lesions of liver. Clinical presentation and imaging features of BCA are nonspecific. Morphological examination of the cyst forms the basis of confirmation of diagnosis of benign cystadenoma and can rule out its malignant transformation to cyctadenocarcinoma. The mainstay of treatment is complete resection of the lesion with conservation of surrounding hepatic parenchyma. We report a case of 59-year-old female patient with a large biliary cystadenoma in whom clinicoradiological findings were nonspecific. Histopathological examination of the cyst wall could establish the final diagnosis.
\end{abstract}

\section{Introduction}

Biliary cyst adenoma (BCA) is a rare, slow growing, benign cystic lesion of liver and account for less than $5 \%$ of solitary cystic lesions of liver [1]. Clinically the patients most often present with abdominal discomfort, pain and sometimes may be asymptomatic [2]. Although $\mathrm{BCA}$ is a benign tumour, the main concern is its high potential for malignant transformation, tendency to recur especially in cases with incomplete excision and difficulty to differentiate cyst adenoma and cyst adenocarcinoma preoperatively. With variability in clinical presentation and imaging data, histopathologic evaluation of the surgically excised cyst forms the gold standard for diagnosis of this rare tumour [2,3]. First described in 1943, there are less than 200 cases reported in recent literature [4].

\section{Case report}

A 59-years-old postmenopausal female of Asian ethnicity reported to the general surgery outpatient department of a Tertiary health care centre in Central India with complaints of slowly enlarging abdominal mass. The mass was noticed by her six months ago and was associated with recurrent episodes of intermittent pain in the right upper quadrant. Anorexia and epigastric discomfort were present for two months. There was no history of trauma, fever, jaundice or weight loss. She had not suffered any major illness in the past. Her personal and obstetrical history was not significant.

The patient was examined as per standard protocols. On general examination there was a large abdominal mass in the right hypochondriac region, firm, slightly tender, extending to midline and reaching up to the right lumbar region. Other systemic examinations were normal.

Initial ultrasound examination (USG) of the abdomen revealed multiloculated cystic lesion of size $15 \times 10 \times 8 \mathrm{~cm}$ containing $100 \mathrm{cc}$ clear fluid in the epigastric area in relation to left lobe of liver and gallbladder fossa. Contrast - enhanced Computerised tomography (CECT) scan of abdomen revealed large $18 \times 14 \times 11 \mathrm{~cm}$, well defined, non-enhancing hypo dense lesion predominantly in the sub capsular region involving the right and left lobe of liver with relative sparring of VI and VII segments of the right lobe of liver. No evidence of solid component or calcifications were seen. There was no evidence of extra hepatic or intrahepatic biliary radical dilatation (Figure 1). MRI scan of abdomen revealed similar findings with evidence of possible communication of the lesion with left hepatic duct. There was no evidence of communication between the lesion and portal venous system.

On further investigations, the full blood counts and serum liver function tests were within normal limits. The cyst fluid aspirated under ultrasonography guidance was clear, reddish brown in colour. Cytological examination of the fluid was negative for malignant cells and there was no evidence of organisms.

The patient underwent diagnostic laparoscopy and the tissue was sent for histopathological examination. On gross examination, two small greyish white cystic tissues pieces aggregating to size $3 \times$ $2 \times 1.5 \mathrm{~cm}$ were received which were entirely subjected to paraffin embedding and processing. On microscopic examination the $\mathrm{H}$ and $\mathrm{E}$ sections revealed variable sized cysts lined by single layer of cuboidal to columnar mucin secreting epithelium resembling biliary epithelium. The cyst wall lining was supported by fibro collagenous stroma (Figure 2A). There was no evidence of multilayering, loss of nuclear polarity, nuclear pleomorphisim or mitosis (Figure 2B-2C). Histological features did not favour commonly occurring post traumatic, parasitic or other hepatic cystic lesions. Taking into consideration, the clinical course, radiological findings and histological features the diagnosis of biliary cyst adenoma was given. Complete resection was advised.

Due to non-availability of patient's consent for further surgical intervention, the complete excision of the cyst could not be performed.

${ }^{\star}$ Correspondence to: Vrushali Vikas Shroff, Department of Pathology, Government Medical College and Hospital, NAGPUR- 440009, Maharashtra, India, Tel: 9930240287; E-mail: vrushalishroff@gmail.com

Key words: cannabidiol, CBD, cannabinoids, delta-9-tetrahydrocannabinol, THC, glioblastoma

Received: November 14, 2018; Accepted: November 27, 2018; Published: November 30, 2018 


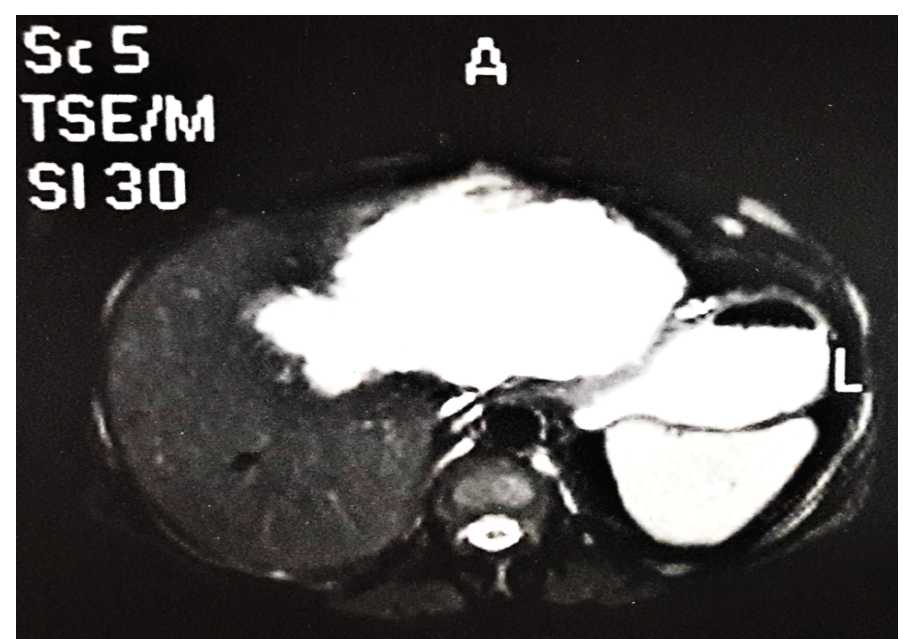

Figure 1. Contrast- enhanced CT SCAN showing large well-defined cystic lesion in liver with no evidence of calcification or solid component

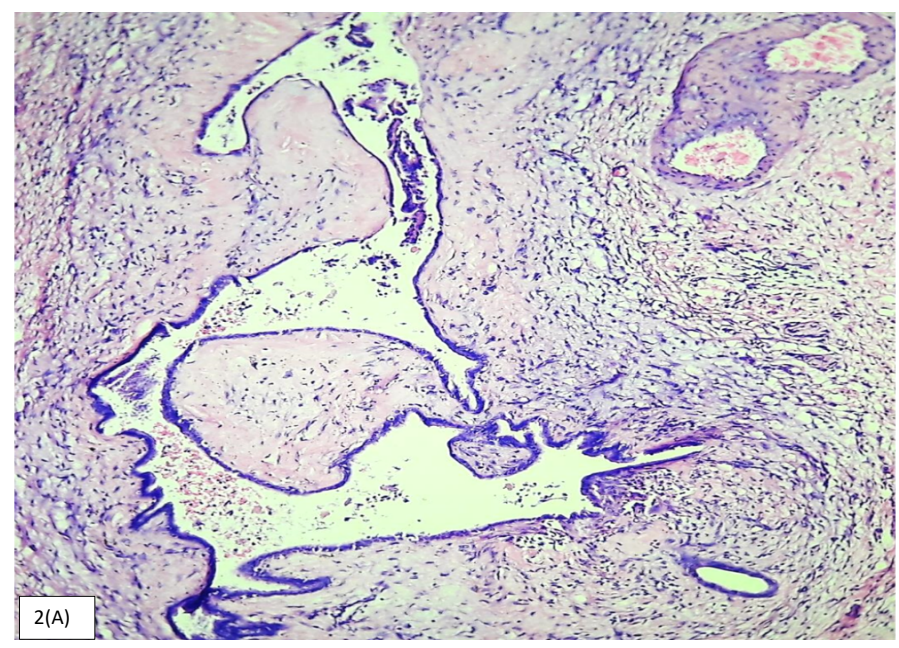

Figure 2A. Photomicrograph showing cyst lined by cuboidal epithelium resembling biliary epithelium with surrounding stroma. (H\&E, 40X)

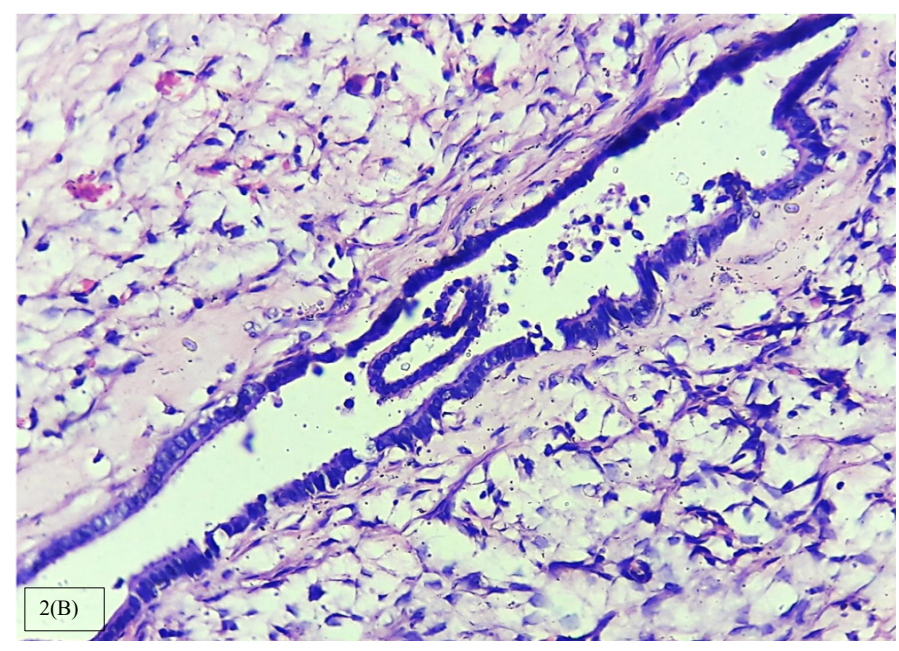

Figure 2B. Photomicrographs showing cyst lined by single layer of cuboidal epithelium with basally placed nuclei. Absence of multilayering, nuclear atypia or mitosis. (H\&E, $40 \mathrm{X}$ )
During follow up at six months, all parameters appeared stable. The patient remains on lifelong clinicoradiological follow up.

\section{Discussion}

Biliary cystadenomas are rare, slow growing, multiloculated, benign cystic lesions of liver with majority of the cases being intrahepatic $(80-85 \%)[5,6]$. These lesions constitute less than $5 \%$ of solitary nonparasitic cystic neoplasms [7]. They predominantly arise in the right lobe of liver (50-55\%) with remaining seen in left lobe of liver and around $15-20 \%$ cases seen arise in both the lobes [8]. The tumours grow slowly reaching a maximum size of $30 \mathrm{~cm}$ in diameter over a few years' time [2]. The highest incidence is seen in middle aged women with mean age of 50 years, with incidence rate of $4: 1$ with respect to males [1].

The origin of these lesions is either direct from the primitive hepatobiliary stem cell or aberrant bile duct and the location is predominantly intrahepatic (80-85\%) with few being extra hepatic [3]. The exact cause of cystadenomas is not known [4]. Some reports have suggested that presence of mesenchymal stroma suggests that the tumour may be arising from ectopic embryonal tissue forming gall bladder or sequestration of embryonic rests in liver. Other studies have suggested the possibility of hormonal pathogenesis for these cystic lesions with mesenchymal stroma as the stromal cells exhibit myofibroblastic phenotype expressing estrogen and progesterone receptors [8]. A few reports have suggested a possible hormonal dependence of cysadenoma with mesenchymal stroma. There are a few reports of this tumour occurring in oral contraceptive users suggesting estrogen containing contraceptive pills may serve as promoters [5].

Clinically the patients are mostly asymptomatic with the lesions detected only incidentally on radiographic imaging. In symptomatic cases the clinical symptoms are nonspecific with abdominal pain in right upper quadrant being the most common complaint. Other symptoms include abdominal distension, nausea, vomiting, palpable abdominal mass. Rarely these lesions show complications like bleeding, infection, rupture, venous obstruction [8]. Biliary obstruction has been reported in $35 \%$ cases either due to tumour or mucin secretion manifesting as jaundice, biliary colic, cholangitis and pruritus [2].

Most widely used radiological investigations are ultrasonography and computerised tomography that allow to study the cyst walls, intracystic projections and possible multilocular arrangement [5]. On gross the lesions vary in size and show smooth, globular external surface with multiple cysts bulging out. The cut surface shows thin walled cysts of varying sizes with smooth lining mostly containing mucinous contents. The inner lining may show presence of papillary projections measuring up to $2 \mathrm{~cm}$ in diameter [4]. Multilocularity is important distinguishing feature that helps differentiating cyst adenomas from various other developmental cystic lesions in liver [2]. The cyst walls may show presence of calcifications or dense fibrosis [6]. There is usually no communication with bile ducts, the presence indicates intraductal papillary mucinous neoplasm (IPMN) rather than cystadenomas [8].

\section{Microscopic examination}

The wall of cystadenomas consists of a cyst lining of biliary epithelium, cellular stroma and dense collagenous connective tissue. The cyst wall lining comprising of the biliary epithelium consists of 
cuboidal to columnar mucin secreting epithelial cells with papillary projections, pale eosinophilic cytoplasm and basally placed nuclei. The features indicating potential malignant changes are nuclear hyperchromasia with enlargement, loss of polarity, multilayering and increased mitosis. The features indicating malignant transformation are stromal or capsular invasion, severe architectural atypia, anaplasia [2].

BCAs are classified into three groups based on the presence dense cellular spindle cell stroma known as mesenchymal stroma into:

1. Hepatobiliary cystadenoma with mesenchymal stroma (HCMS)

2. Hepatobiliary cystadenoma without mesenchymal stroma

3. HCMS with polypoidal epithelial component projecting into cyst lumen [7].

Hepatobiliary cystadenoma with mesenchymal stroma usually occurs in female in fifth decade of life and is regarded as precancerous lesion [9]. Cystadenomas without mesenchymal but with hyaline stroma occurs in both men and women in older age group and seem to undergo frequent malignant transformation. The present case appears to belong to the second category. The histological features which help in differentiating the cystadenomas from other cystic lesions of liver are the columnar epithelium, papillary infoldings, mesenchymal or ovarian like stroma [2].

Preoperative determination of serum and cyst fluid CEA and CA19-9 levels in the cyst fluid can helps to differentiate cyst adenoma from other cysts and cyst adenocarcinoma $[5,10]$.

Similar to lesions seen in pancreas and ovary, two histological variants of biliary cystadenoma are identified - mucinous and serous. The mucinous type is commoner, seen more predominantly in middle aged females and associated with mesenchymal stroma whereas the serous type is rarer and not known to undergo malignant transformation. It is crucial to differentiate cystic lesions of liver as cystadenomas has high tendency to recur with a potential for malignancy [2].

The most common modality of treatment is surgical excision whenever possible due to tendency of lesion for malignant transformation. Patient follow up is essential to monitor recurrences, malignant transformation and postsurgical complications $[6,10]$.

\section{Conclusion}

In view of nonspecific clinicoradiological features, histopathological examination of the cyst forms mainstay for specific diagnosis of biliary cystadenoma. Differentiation of biliary cystadenomas from other cystic lesions of liver is crucial for management and follow up of the patients.

\section{Acknowledgement}

Dr. Waman Krishnaji Raut, Professor and Head (Department of Pathology), Government Medical College and Hospital, Nagpur 440009, Maharashtra, India.

\section{References}

1. Joel JM, Jeyasingh SD, Kalyanaraman S (2016) Biliary Cystadenoma: A Case Report. J Clin Diagn Res 10: ED19-20. [Crossref]

2. Manouras A, Markogiannakis H, Lagoudianakis E, Katergiannakis V (2006) Biliary cystadenoma with mesenchymal stroma: report of a case and review of the literature. World J Gastroenterol 12: 6062-6069. [Crossref]

3. Ahanatha Pillai S, Velayutham V, Perumal S, Ulagendra Perumal S, Lakshmanan A et al. (2012) Biliary cystadenomas: a case for complete resection. HPB Surg 2012: 501705. [Crossref]

4. Ishak KG, Willis GW, Cummins SD, Bullock AA (1977) Biliary cystadenoma and cystadenocarcinoma. Report of 14 cases and review of the literature. Cancer 39: 322338. [Crossref]

5. Bartolome MAH, Ruiz SF, Romero IM, Lojo BR, Prieto IR, et al. (2009) Biliary cystadenoma. World J Gastroenterol 15: p.3573.

6. Soares KC, Arnaoutakis DJ, Kamel I, Anders R, Adams RB, et al. (2014) Cystic neoplasms of the liver: biliary cystadenoma and cystadenocarcinoma. J Am Coll Surg 218: pp.119-128. [Crossref]

7. Koroglu M, Akhan O, Akpinar E, Oto A, Gumus B (2006) Biliary cystadenoma and cystadenocarcinoma: two rare cystic liver lesions. JBR-BTR 89: 261. [Crossref]

8. Al-Qahtani HH (2016) Diagnostic uncertainty of hepatobiliary cystadenoma: Report of 11 cases and review of the literature. Journal of Taibah University Medical Sciences 11: $19-25$.

9. Myers JL (2017) Rosai and Ackermans Surgical Pathology. (11 $1^{\text {th }}$ Edn): 822-823.

10. Lee CW, Tsai HI, Lin YS, Wu TH, Yu MC, et al. (2015) Intrahepatic biliary mucinous cystic neoplasms: clinicoradiological characteristics and surgical results. $B M C$ Gastroenterol 15: 67. [Crossref]

Copyright: (C2018 Shroff VV. This is an open-access article distributed under the terms of the Creative Commons Attribution License, which permits unrestricted use, distribution, and reproduction in any medium, provided the original author and source are credited. 